\title{
Paul Dudley White International Lecture: Cardiac Performance as Viewed Through the Pressure-Volume Window
}

\author{
Hiroyuki SugA, M.D., D.Med.Sc.
}

\begin{abstract}
SUMmaRY
Viewing canine left ventricular performance through the pressure-volume (P-V) window, I proposed a new index of ventricular contractility (Emax: endsystolic $\mathrm{P}-\mathrm{V}$ ratio or maximum elastance) in my doctoral thesis at the University of Tokyo in 1969. After I joined Dr. Kiichi Sagawa (deceased in 1989) at Johns Hopkins University, we firmly established the Emax concept during 19711978. The concept was extended to derive the systolic P-V area (PVA) as a new measure of the total mechanical energy generated by ventricular contraction in 1978. Experiments have revealed that PVA closely correlates with cardiac oxygen $\left(\mathrm{O}_{2}\right)$ consumption $\left(\mathrm{Vo}_{2}\right)$ under various loading conditions at a constant Emax, the $\mathrm{VO}_{2}$-PVA relation changes its elevation with Emax, and the $\mathrm{O}_{2}$ costs of PVA and Emax characterize the mechanoenergetics of cardiac contraction under various normal and abnormal conditions in an innovative manner (Suga: Physiol Rev 70: 247-277, 1990). Emax and PVA can also evaluate the ventriculo-arterial hydraulic and energetic matching in normal and failing hearts. Emax and PVA have thus widely opened the P-V window to the extent that human and animal normal and failing cardiac performance can be characterized in a physiologically sound manner. (Jpn Heart J 35: 263-280, 1994)
\end{abstract}

Key words: Emax Time-varying elastance PVA Pressure-volume area Mechanoenergetics

$\mathrm{M}$ ANY of you might doubt your eyes when you first look at the title of my lecture and are puzzled why pressure and volume are the current topics. You may think that this is 1993, not 1893! The famous Otto Frank $^{1)}$ is not present! The famous Ernest Henry Starling ${ }^{2)}$ is not present, either. Is there any recent discovery of genes expressing pressure and volume? No, that

From the Department of Physiology II, Okayama University Medical School, Okayama, Japan.

This named lecture was established in 1966 by the American Heart Association (AHA) after an eminent American physician Dr. Paul Dudley White for his leadership in the advancement of cardiology throughout the world. The lecturers have been elected as honorary fellows of the AHA Council on Clinical Cardiology. This, the 17th lecture, was given at the AHA 66th Scientific Session on November 9, 1993 in Atlanta, Georgia, USA. The abstract of the lecture was published in Circulation 88 (Suppl Part 2): I-C, 1993. This paper, which includes figures, is a slightly condensed version of the $45 \mathrm{~min}$ lecture.

Address for correspondence: Prof. Hiroyuki Suga, Department of Physiology II, Okayama University Medical School, 2 Shikatacho, Okayama 700, Japan. 
is not the case. Instead, I am going to review progress in our understanding of the ventricular $\mathrm{P}-\mathrm{V}$ relationship over the past quarter century. ${ }^{3)}$

I am going to explain why the P-V relationship is very meaningful and important in today's cardiovascular physiology and cardiology. I hope that you will realize the power of pressure and volume variables for better understanding the performance of normal and diseased hearts.

\section{Historical background}

As shown in Figure 1, nearly a century ago, the German physiologist Otto Frank characterized ventricular contraction in a P.V diagram. ${ }^{4}$ Panel A displays a P-V loop of a contraction, together with the end-diastolic and end-systolic P-V curves. He used the frog heart, which has an end-systolic P-V relationship that is both markedly nonlinear and sensitive to afterload conditions. The number of medical physiology textbooks that still show Frank's diagram has decreased ${ }^{5)}$ and major textbooks have already adopted the P-V relationship which has been established in mammalian hearts over the past two decades. ${ }^{6}$

Nearly 70 years ago, a British physiologist Ernest Henry Starling extended Frank's concept of the ventricular P-V relationship to a canine pumping heart and studied the cardiac output-filling pressure relationship, as shown in Figure 1, Pancl B. You are familiar with Starling's law of the heart, ${ }^{2)}$ which is useful in

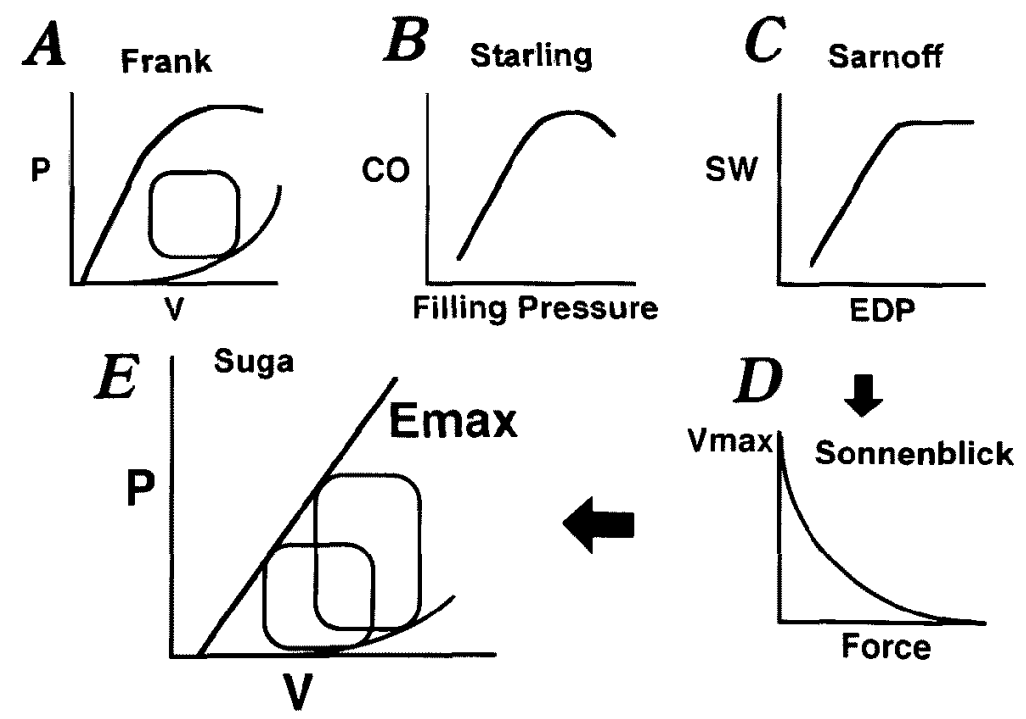

Figure 1. History of views on cardiac performance. Frank: Frank's pressure-volume (P-V) relationship. Starling: Starling's cardiac output (CO) curve as a function of filling pressure. Sarnoff: Sarnoff's ventricular function curve relating stroke work (SW) to end-diastolic pressure (EDP) of the ventricle. Sonnenblick: Sonnenblick's force-velocity curve with Vmax as an index of contractility. Suga: Emax as the slope of the endsystolic $\mathrm{P}-\mathrm{V}$ relation. 
cardiology. Starling's cardiac output curve and its modifications, such as Sarnoff's ventricular function curve (Figure 1, Panel C), ${ }^{7}$ are categorized into this approach. Using these diagrams, inotropism or contractility changes can be visualized as a shift of the function curve. ${ }^{7)}$

The heart can also be viewed as an assemblage of myocardial fibers and characterized by the shortening velocity-force relationship (Figure 1, Panel D). This approach is analogous to the approach used for skeletal muscles, which were analyzed beautifully 50 years ago by A.V. Hill. ${ }^{8)}$ About 30 years ago, when I was a medical student, Edmund Sonnenblick ${ }^{3)}$ established Vmax as an index of contractility (Figure 1, Panel D). Vmax is the maximum unloaded shortening velocity. The Vmax concept has been extended to ventricular circumferential shortening velocity ( $\mathrm{Cl}$ ) for clinical use and is still used in cardiology. ${ }^{10}$

When I started my doctoral research career in the late $1960 \mathrm{~s}$, I had somc difficulty in accepting the Vmax concept for cardiac muscle, because, unlike skeletal muscles, cardiac muscle does not normally contract in tetanus. I had a feeling that the characterization of ventricular contractile behavior was necessary for a better understanding of normal and diseased cardiac performance. Some of my colleagues said it was obsolete to study again the P-V relationship in the days of Vmax. This kind of comment discouraged me initially, but I was soon revived by my strong intuition that there must be some undiscovered concepts in the P. $\mathrm{V}$ diagram.

Then, one day, very fortunately, the central idea for exploring Emax occurred suddenly while I was serving once a week as a night dispensary doctor. Thereafter, Emax became the theme of my doctoral thesis in Tokyo. "1) Emax is the key word in today's talk. Emax (or interchangeably Ees) denotes the slope of a linear end-systolic P-V relation, as shown in Figure 1, Panel E, ${ }^{12)}$ reopened the P-V window, which had been almost closed in those days. Since then, Emax has greatly facilitated our understanding of cardiac performance. ${ }^{13\rangle}$

\section{Advantages of pressure-volume window}

Pressure and volume are the two most fundamental physical variables with which to describe the mechanical properties of any chamber, although the ventricle is actively contracting unlike ordinary manufactured chambers. The product of pressure and volume gives mechanical work. Cardiac mechanical work is given by the area within a P-V loop drawn in the P-V diagram (Figure 2, Panel A); this is well known from the fact that the P-V diagram used to be called the work diagram. ${ }^{1)}$ The greater the area within the P-V loop, the greater the work. This is one of the main advantages of using the P-V diagram.

Another advantage of the P-V diagram is that a $\mathrm{P}-\mathrm{V}$ relation curve reflects elastic properties of the chamber (Figure 2, Panel B). The steeper the curve, the 

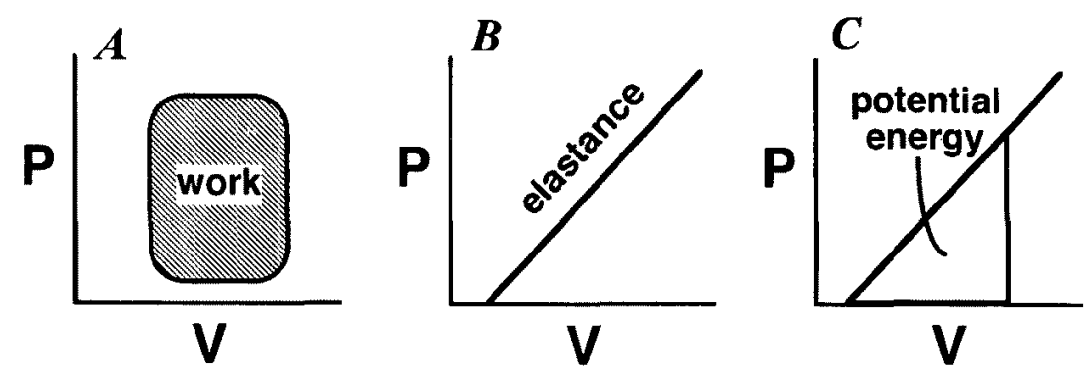

Figure 2. Information contained in the pressure-volume (P-V) window. A: Mechanical work is given by the area within a P-V loop. B: Volume elastance is given by the slope of a P-V relation. C: Elastic potential energy is given by the area under a P$\mathrm{V}$ relation.

stiffer (or the more elastic) the chamber. Therefore, a P-V relation indicates the elastance of the chamber. When the P-V relation changes with time, it means that the chamber's elastance changes with time; this property is termed a timevarying elastance. ${ }^{12)}$ This is the basis of my discovery of the Emax concept. ${ }^{12)}$

Theoretically, the area under a P-V relation represents mechanical potential energy (Figure 2, Panel C). This is the third major advantage of using the P$\mathrm{V}$ diagram, and it is the basis of my discovery of the cardiac energy concept in terms of PVA. ${ }^{14)}$

In these respects, the P-V diagram provides a better characterization of ventricular performance than Starling's cardiac output-filling pressure diagram,2) Sarnoff's stroke work-end-diastolic pressure diagram ${ }^{7)}$ and Sonnenblick's Vmaxforce diagram. ${ }^{\text {) }}$

Although the volume and pressure variables are global, they are vector sums of the lengths of all the sarcomeres and the forces generated by crossbridges $(\mathrm{CBs})$ within the wall of the ventricle. Figure 3 shows the correspondence of the $\mathrm{P}-\mathrm{V}$ diagram to the sarcomere and CB behavior. Ventricular filling produces the horizontal P-V segment. During this period, sarcomeres are stretched and the number of available CBs increase, providing the basis of the Starling effect. Excitation causes contraction and the number of attached CBs increases, developing force and pressure, and forming the isovolumic contraction segment of the P-V loop. During ejection, attached CBs turn over and slide, producing external work and creating the ejection segment of the loop. During relaxation, the number of attached GBs and henceforce decrease.

The area within the P-V loop is external work. This work is equal to the integrated work that all CBs produce, according to the law of energy conservation.

Thus, although pressure and volume are global variables, they are closely related to $\mathrm{CB}$ behavior. Although their detailed relationships still remain to be elucidated, the P-V window has a fourth advantage: it contains information on 


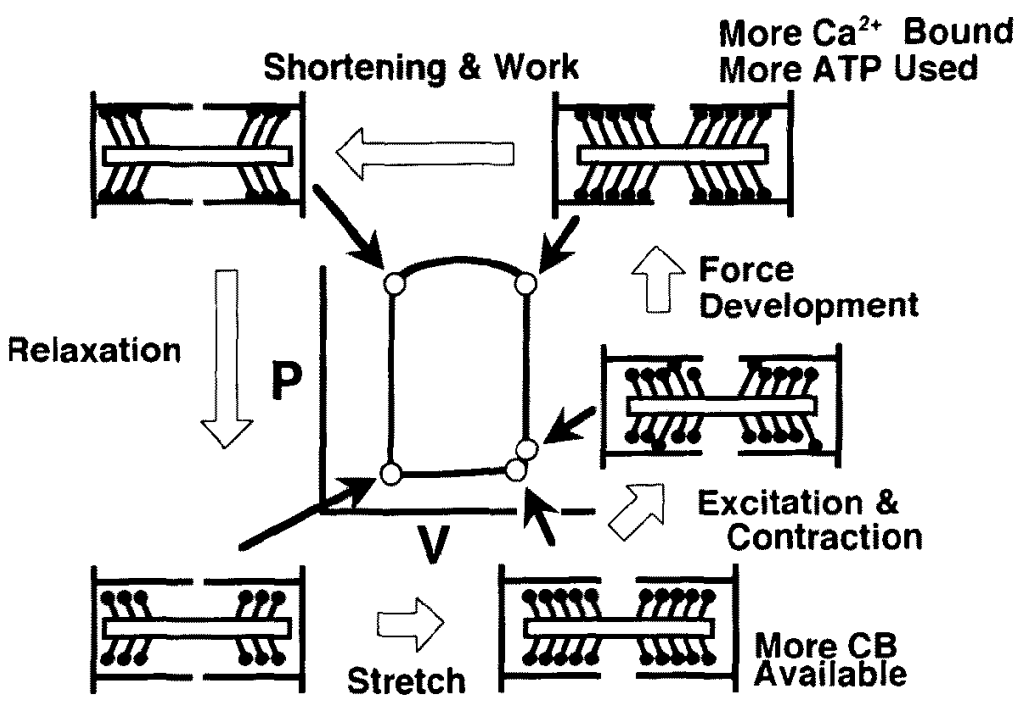

Figure 3. Correspondence of the P-V diagram to sarcomere and crossbridge (CB) behavior. Sarcomere length increases with volume. The number of attached $\mathrm{CB}$ incrcases with contraction. Attached CB is shown by a myosin head (solid circle) on a thin filament.

the mechanoenergetically integrated $\mathrm{CB}$ behavior within the chamber wall. This advantage has reinforced the basis of the PVA concept. ${ }^{3,14]}$

\section{Experimental outcome}

When I started to study the P-V relationship of the canine heart in Japan in 1967, the P-V diagram had been used occasionally only for quantifying heart size, the ejection fraction and external mechanical work. The power of the P-V diagram in revealing various mechanical and energetic aspects of cardiac performance was not suspected. However, I strongly believed that there must be something new and important undiscovered in the P-V diagram.

Figure 4 is taken from my doctoral thesis published in 1969. ${ }^{11}$ The left side shows left ventricular P-V loops of an in situ canine heart after cutting cardiac sympathetic nerves. Ventricular volume was measured by combining an electromagnetic flowmeter on the aorta and an indicator dilution technique. Therefore, there was some uncertainty regarding the absolute volume.

Nevertheless, the data revealed that the end-systolic P.V points of these P$\mathrm{V}$ loops fell on a diagonal linc. The $\mathrm{P}-\mathrm{V}$ points at a given mid-systolic time also fell on a less steeper line. The slope of such a P-V line increased to the endsystolic peak value, Emax (initially termed $\mathbf{e}_{\max }$ ), and then fell to the diastolic lcvel in each cardiac cycle. ${ }^{11)}$ The curves on the right side of Figure 4 show that the slope changes $e(t)$ of these P-V relations and Emax (peak of e) of these 3 contractions under different loading conditions are similar. These curves marked the first 


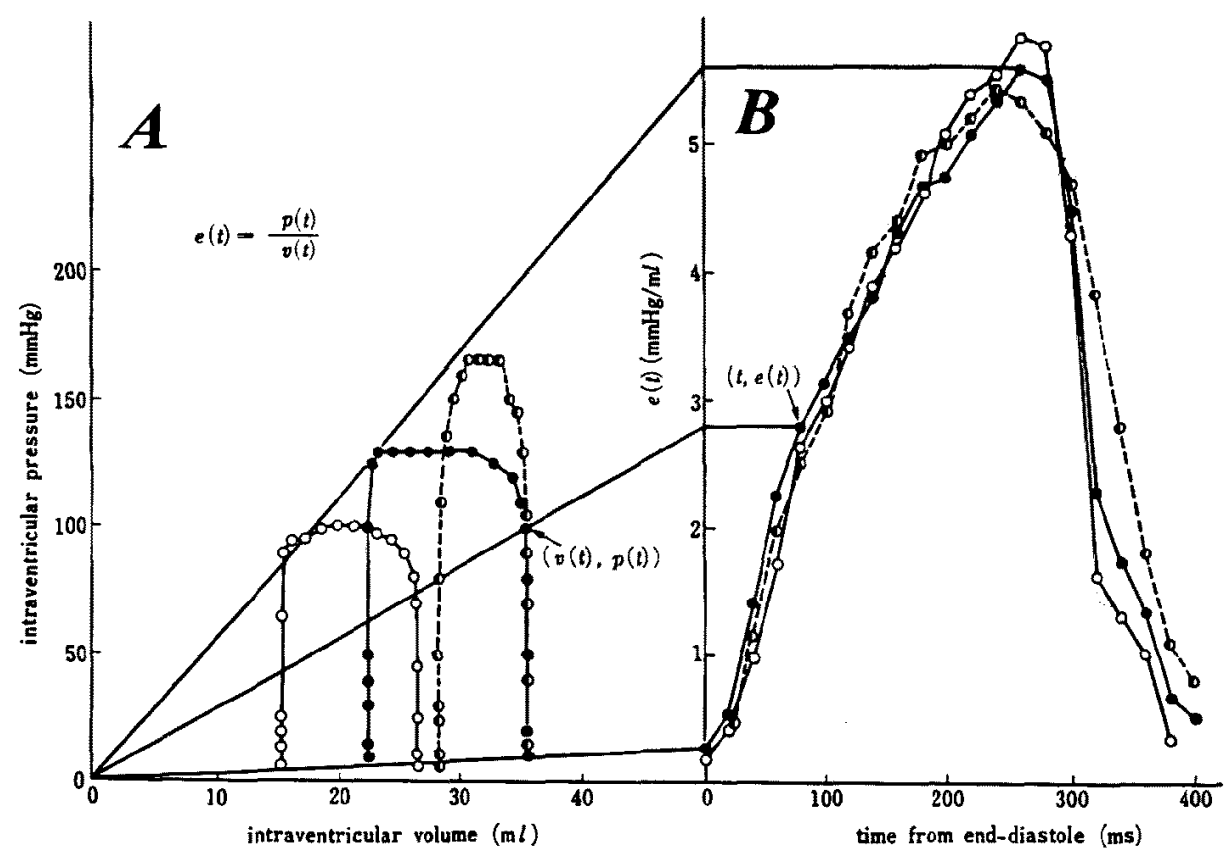

Figure 4. Left ventricular $P-V$ loops of an in situ canine heart $(A)$ and the slope changes of the P-V relations as a function of time under different loading conditions in a stable contractile state (B). (Modified from Figure 2 of Ref 11).

characterization of the time-varying elastic nature of a beating and ejecting ventricle. ${ }^{(1)}$

\section{Pressure-volume loops and Emax}

Figure 5 is also taken from my thesis. ${ }^{11)}$ This shows that cardiac sympathetic nerve stimulation shifted the P-V loops upward and leftward. As the result, the slope of the end-systolic P-V relation became steeper and its slope Emax also increased markedly. Thus, Emax was suggested as a new index of ventricular contractility.

After I obtained a Doctorate of Medical Sciences at the University of Tokyo, I joined Dr. Kuichi Sagawa at Johns Hopkins University. He was intrigued by the Emax concept and encouraged me to develop the concept. I stayed with him for nearly six years in the 1970s, producing several original papers that established the Emax concept. ${ }^{12,15-17)}$ Our two Circulation Research papers ${ }^{12,15)}$ have been cited more than 1100 times and the total number of citations of my more than 100 papers on Emax and the P-V window numbers more than 3000 , according to a recent Science Citation Index survey. Without Dr. Sagawa, I probably would not be speaking at this lectern.

In Baltimore, we confirmed the validity of Emax in canine hearts by im- 


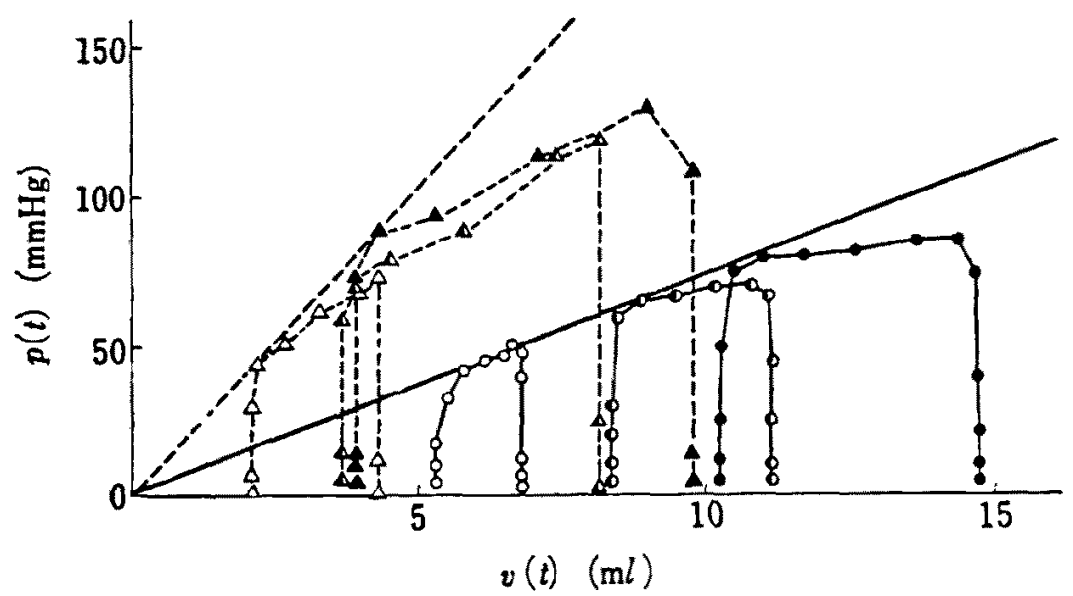

Figure 5. Left ventricular P-V loops under control (circles and solid lines) and cardiac sympathetic nerve stimulation with an augmented slope of the end-systolic P$\checkmark$ relation (triangles and dotted lines). (Modified from Fig. 4 of Ref 11 ).
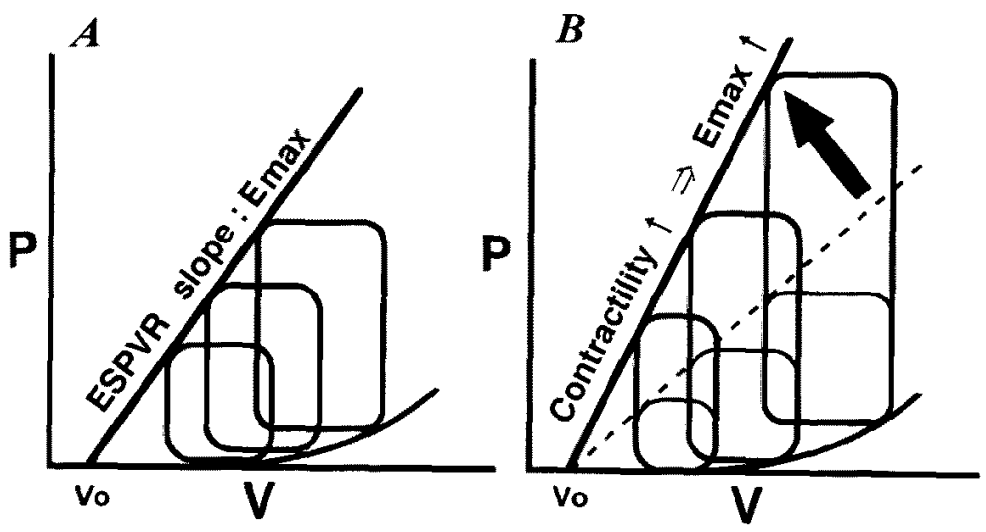

Figure 6. Schematic drawing of $\mathrm{P}-\mathrm{V}$ loops and the end-systolic P-V relation (ESPVR) in control (A) and an enhanced contractile state (B). The slope of the ESPVR line is Emax. Emax is largely independent of preload and afterload. The end-systolic P. $V$ line has a positive volume intercept $V o$.

proving ventricular volumetric methods. ${ }^{12,18)}$ Figure 6 schematically summarizes representative P-V loops and the end-systolic P-V relation (ESPVR) in a stable contractile state (Panel A). We confirmed that Emax was largely independent of preload and afterload by refined measurements and load control. In addition, we discovered that the end-systolic P-V line had a positive volume intercept Vo (which was initially called Vd).

When ventricular contractility was enhanced by administration of epinephrine, we confirmed that the P-V loop grew markedly during positive inotropism. ${ }^{12,15)}$ The end-systolic P-V relation became steeper and Emax in- 
creased (Figure 6, Panel B) in a similar manner to Figure 5, but Vo was unaffected. We obtained similar results with other positive inotropic interventions. ${ }^{13)}$ Negative inotropic interventions produced the opposite results. ${ }^{13)}$ Thus, it became our contention that Emax, defined as the end-systolic maximum elastance of the ventricle, could indicate the contractile state of the chamber. ${ }^{12,13,15)}$

\section{Limitations}

However, as more investigators in various laboratories studied Emax experimentally and clinically, they encountered various problems. Some are fundamental and others are practical and clinical. Emax is not an exception to "Murphy's law" that "Every Solution Breeds New Problems." One main limitation is that Emax is slightly dependent on loading conditions. For example, isovolumic Emax may be different from ejecting Emax depending on ejection fraction. ${ }^{19)}$ In addition the end-systolic P-V relation is not always linear. For example, it tends to be concave downward at a high Emax and upward at a low Emax. ${ }^{20)}$ Finally, normalization of Emax for heart size is not simple. ${ }^{21)}$ These are very interesting issues, but they are beyond the scope of this lecture.

\section{Clinical application}

After Emax was publicized by our 1973 and 1974 Girculation Research papers, ${ }^{2,15)}$ cardiologists began to apply Emax to patient care. The first cardiologist was Myron Wcisfeldt at Johns Hopkins. He presented his work, under the title of "Emax as a new contractility index in man", at the 1976 meeting of the AHA. ${ }^{22)}$ The second one was William Grossman at Harvard. ${ }^{23)}$ End-systolic P-V relations were compared in three patient groups with normal and moderately and severely failing hearts. Evidently, the Emax line shifted to the right as cardiac failure became severe. Many similar results in patients have been reported by different groups over 15 years and Emax has helped cardiologists assess the degree of heart failure. After Jan Baan developed a volumetric conductance catheter in $1980,{ }^{24)}$ ventricular volumetry became much easier in both animal experiments and clinical settings. ${ }^{25)}$

\section{Discovery of PVA}

When I started my research career, I was taught the adage: "Make your own work important." This motto motivated me to expand the Emax concept beyond an index of contractility. This extension of the framework for Emax, first developed in 1977, resulted in the PVA concept. ${ }^{14)}$

The contracting ventricle has a time-varying elastic nature (Figure 7, Panels $\mathrm{A}$ and $\mathrm{B}$ ). The increasing slope of the $\mathrm{P}-\mathrm{V}$ relation and hence the increasing elastance during systole, is symbolized by a thickening spring within the ventricu- 


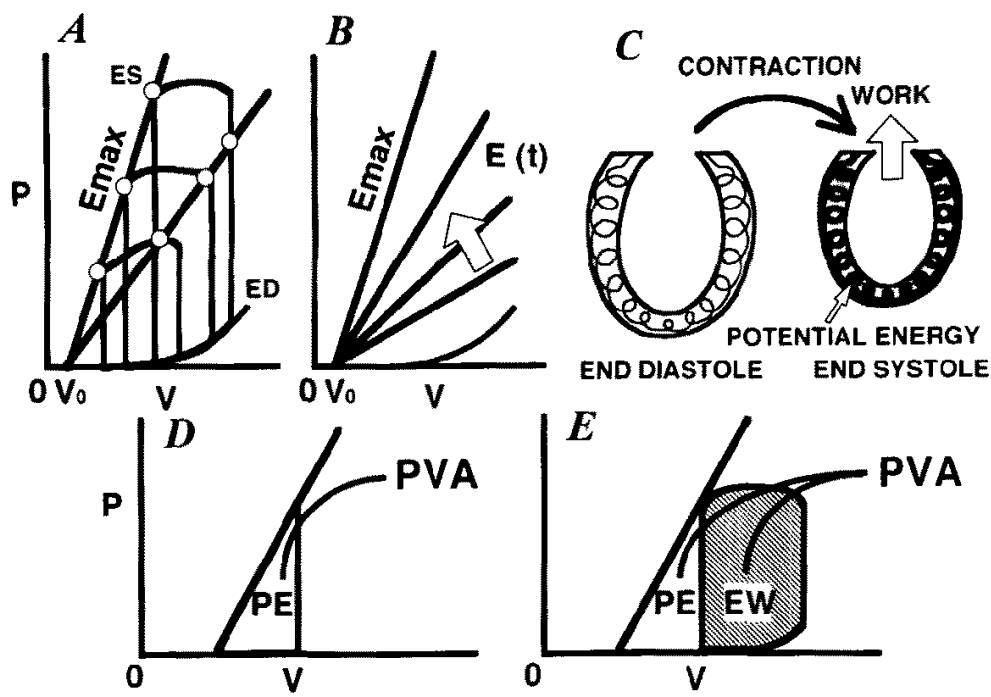

Figure 7. The time-varying P-V relation (A and $B$ ) and time-varying elastance model $(C)$ of the ventricle. The increasing slope of the P.V relation and hence the increasing elastance during systole are symbolized by a thickening spring within the ventricular wall as shown in Panel $C$. This varying-elastic spring is an analogy to the increasing number of attached CBs within the ventricular wall during contraction. Each attached CB has elasticity and the overall elasticity is considered proportional to the total number of attached CBs although the detailed relation remains to be determined.

The new idea form from the time-varying elastance model of the heart is that mechanical energy must be increased within the time-varying elastance when the elastance increases within the ventricular wall. This is analogous to the elastic energy stored in a stretched spring. Physics tells us that such an elastic energy can be assessed in the strcss-strain diagram. Therefore, vcntricular clastic energy can be quantified in the P.V diagram.

lar wall (Figure 7, Panels $\mathrm{C}$ and D). This variable-elastic spring is analogous to the increasing number of attached $\mathrm{CBs}$ within the ventricular wall during contraction. Each attached CB has elasticity and the overall elasticity is assumed to be proportional to the total number of attached CBs.

The new idea was that mechanical energy must be increased within the time-varying elasticity when the elasticity increases within the ventricular wall. ${ }^{14)}$ This is analogous to elastic energy stored in a stretched spring. Physics tells us that such an elastic energy can be assessed in a stress-strain diagram. Therefore, I proposed that ventricular elastic energy can be quantified in the P-V diagram as shown in Figure 7. ${ }^{14}$

Figure 7, Panel E shows an isovolumic contraction. The elastic energy is given theoretically by a triangular area under the Emax line. This is the elastic potential energy generated during systole and stored at the end of systole in the ventricle. Figure 7,'Panel $\mathrm{F}$ shows an ejecting contraction. The elastic energy is 
given by a triangular area under the Emax line. This is also the elastic potential energy generated during systole and stored at end systole in the ventricle. On top of this potential energy, an ejecting ventricle has performed external work by end systole. This work is given by the area within the P-V loop. The sum of the external work and the potential energy is the total mechanical energy. Therefore, the sum of these two areas gives the total mechanical energy that the ventricle generates during each contraction. The isovolumic contraction is a special case where external work is zero. Therefore, I called this specific area "P-V area" and abbreviated it as "PVA". ${ }^{14)}$ Thus, PVA is a new measure of total mechanical energy generated by each contraction of the ventricle. This PVA concept is an epoch-making extension of the Emax concept.

\section{Myocardial ATPases}

Figure 8 shows major energy consuming activities of myocardium. $\mathrm{Most} \mathrm{O}_{2}$ is consumed to produce ATP. ATP is a primary substrate for three ATPases. The first is $\mathrm{Na} / \mathrm{K}$ ATPase, which is related to membrane excitation. The next is $\mathrm{Ca}$ pump ATPase, which is related to Ca handling in the excitation-contraction (EC) coupling. The third is myosin ATPase, which causes CB cycling to develop force, exhibit Emax, elicit shortening, produce PVA and perform work.

We studied the correlation between PVA and myocardial $\mathrm{O}_{2}$ consumption $\left(V_{O_{2}}\right)$, because $\mathrm{VO}_{2}$ is the input to the cardiac bioengine and PVA is the output. Although PVA is onc of the mcchanical measurcs integrating CB mechanics, the law of energy conservation implies that it measures the total mechanical energy

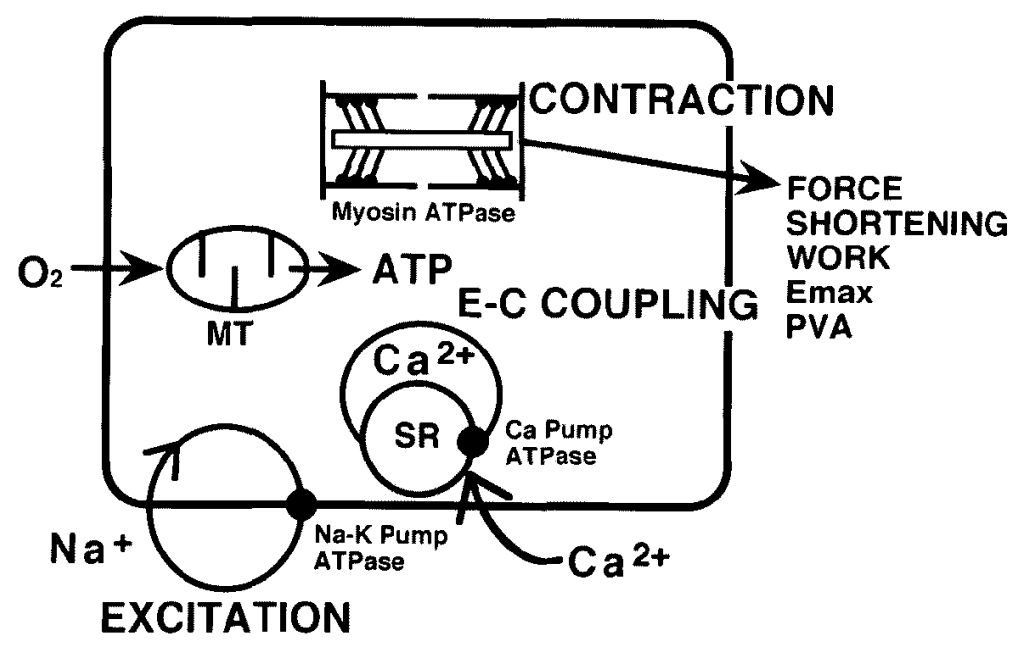

Figure 8. Three major energy consuming processes of myocardium. $\mathrm{MT}=$ mitochondria; $\mathrm{SR}=$ sarcoplasmic reticulum. ATPases are shown by solid circles. 
of CBs.

\section{Cardiac $\mathrm{O}_{2}$ consumption versus PVA}

In our experiments on PVA, we used the same canine heart model as in our Emax studies, except for the addition of an oximeter to measure $\mathrm{Vo}_{2}$. We measured PVA and $\mathrm{Vo}_{2}$ of the left ventricle in a stable contractility, namely at a constant Emax. Figure 9, Panel A shows a linear regression of $\mathrm{Vo}_{2}$ on PVA in isovolumic contractions with increased volume loading. Ejecting contractions produced similar results (Figure 9, Panel B). Superimposing these isovolumic and ejecting results produced Figure 9, Panel C. Both isovolumic and ejecting contractions produced virtually identical $\mathrm{Vo}_{2}$-PVA relationships. Based on these results, we concluded that in a given stable contractility, $\mathrm{VO}_{2}$ is correlated linearly with PVA. ${ }^{2627)}$ We also confirmed this tight relation between $\mathrm{Vo}_{2}$ and PVA under various loading conditions in a stable contractile state. ${ }^{27)}$

We studied the effects of positive inotropism on the $\mathrm{Vo}_{2}$-PVA relation. Epinephrine was given in this casc. Wc found that epincphrinc increascd Emax and simultaneously elevated the $\mathrm{VO}_{2}$-PVA relation in a parallel manner (Figure 10). ${ }^{27,28)}$ We obtained similar results with many positive inotropic agents including various new cardiotonic agents. ${ }^{27)}$ Negative inotropic agents lowered the $\mathrm{Vo}_{2}-$ PVA relation in a parallel manner. ${ }^{27}$ ) These results were confirmed by other investigators.

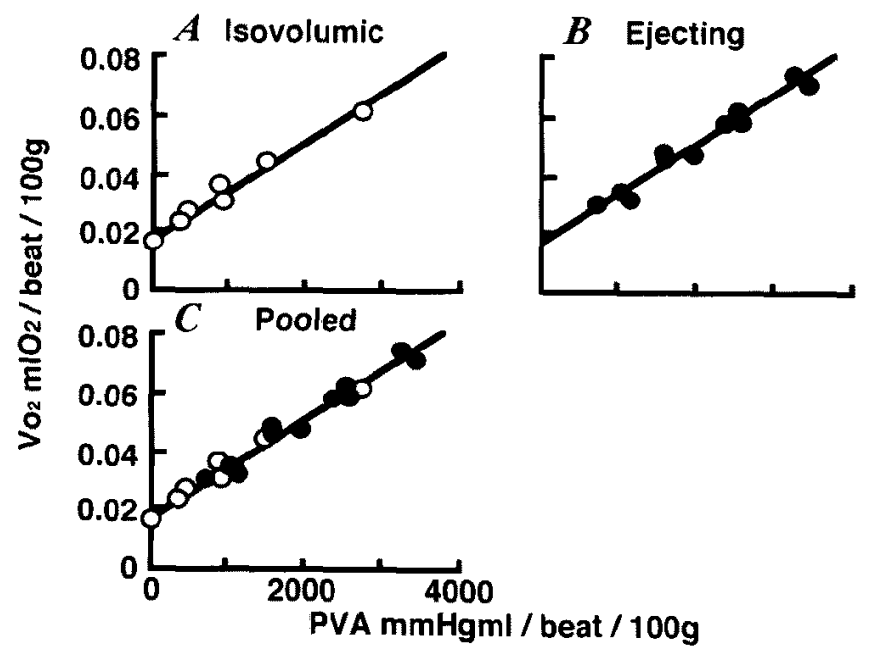

Figure 9. Linear regression of $\mathrm{VO}_{2}$ on $\mathrm{PVA}$ in isovolumic contractions with increased volume loading (Panel A), in ejecting contractions (Panel B), and in both contractions pooled together (Panel C). 


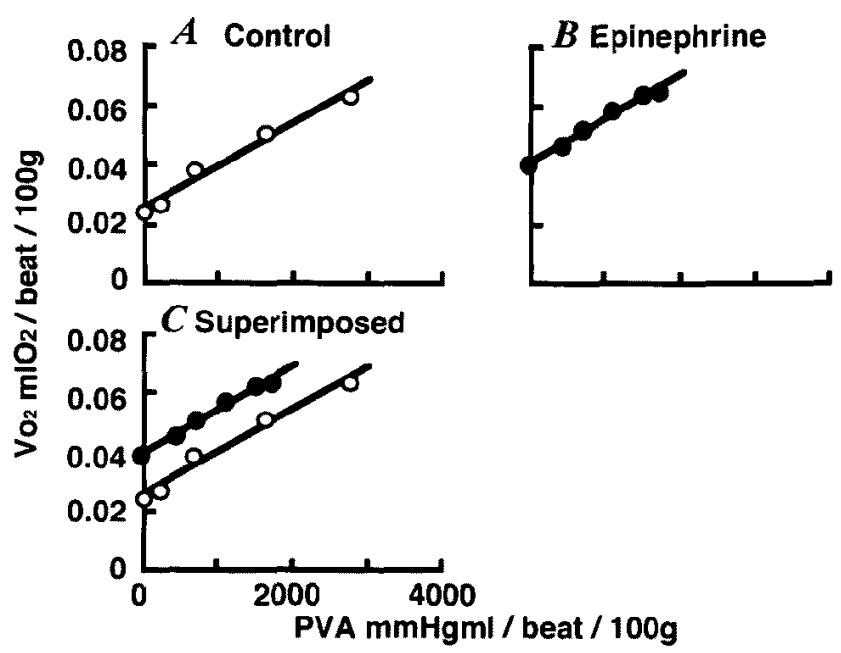

Figure 10. Linear regression of $\mathrm{VO}_{2}$ on PVA in contractions in control contractile state (Panel A) and in contractions in an enhanced contractile state with epinephrine (Panel B). Panel $\mathrm{C}$ is a superimposition of $\mathrm{A}$ and $\mathrm{B}$.
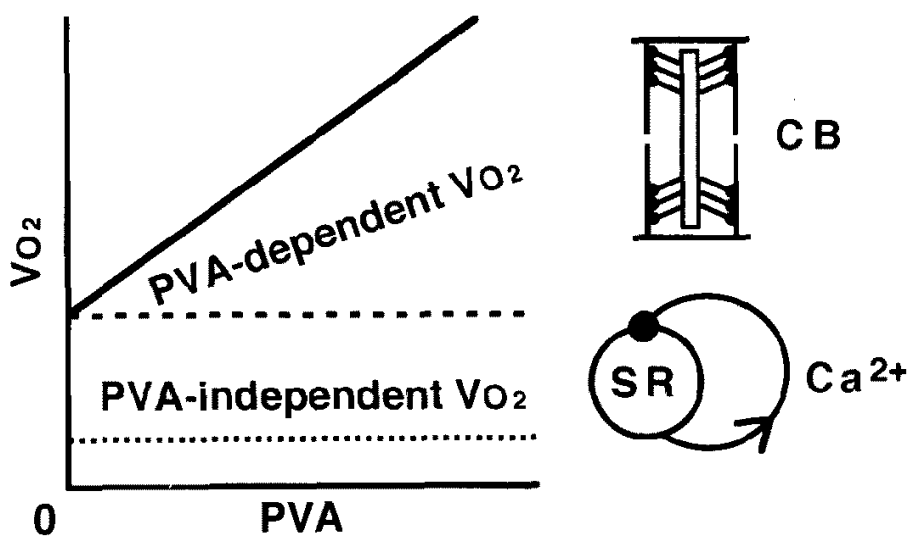

Figure 11. Macro and micro correspondence of PVA-dependent and PVA-independent $\mathrm{Vo}_{2}$, dividing $\mathrm{VO}_{2}$ at the $\mathrm{Vo}_{2}$ intercept. $\mathrm{CB}=$ crossbridges; $\mathrm{SR}=$ sarcoplasmic reticulum.

\section{PVA-independent and PVA-dependent $\mathrm{Vo}_{2}$}

Figure 11 shows schematically how the $\mathrm{Vo}_{2}$-PVA relation at the macro-level corresponds to the micro-level myocardial activities. The $\mathrm{Vo}_{2}$-PVA relation can be divided into two components by a horizontal line at the level of the $\mathrm{VO}_{2}$ intercept at zero PVA. We call the lower fraction of $\mathrm{Vo}_{2}$ "PVA-independent $\mathrm{VO}_{2}$ " because this fraction remains even when PVA is reduced to zero. We call the upper $\mathrm{VO}_{2}$ fraction "PVA-dependent $\mathrm{VO}_{2}$ " because this fraction increases with increases in PVA. We assume that the PVA-independent component is related to 
non-mechanical activity of the myocardium, consisting primarily of $\mathrm{Ca}^{2+}$ ion handling in E-C coupling and basal metabolism. ${ }^{27)}$ We also assume that the PVAdependent component is related to the mechanical activity of myocardium, namely $\mathrm{CB}$ cycling. ${ }^{27)}$ Based on these considerations, we can estimate the effects of inotropic interventions on $\mathrm{Ca}^{2+}$ handling from a change in the elevation of the $\mathrm{VO}_{2}$-PVA relation or its $\mathrm{VO}_{2}$ intercept (=PVA-independent $\left.\mathrm{Vo}_{2}\right){ }^{27)}$

\section{Oxygen costs of PVA and Emax}

Based on these experimental studies, we obtained an empirical equation ${ }^{27,29)}: \mathrm{Vo}_{2}=a \cdot \mathrm{PVA}+b=a \cdot \mathrm{PVA}+c \cdot \mathrm{Emax}+d$. This relates $\mathrm{Vo}_{2}$ to $\mathrm{PVA}$ and Emax. $a \cdot$ PVA corresponds to the PVA-dependent $\mathrm{Vo}_{2}$ and $b$ corresponds to the PVA-independent $\mathrm{VO}_{2}$. Since $b$ changes with Emax, $b$ can be written as the sum of $c \cdot$ Emax $+d$. Then, $a$ indicates the $\mathrm{O}_{2}$ cost of PVA and $c$ indicates the $\mathrm{O}_{2}$ cost of Emax. These concepts are illustrated in Figure 12.

We obtained $a, b, c$ and $d$ values for various positive and negative inotropic interventions. As a result, we found that both the $\mathrm{O}_{2}$ costs of PVA and Emax were stable for most conventional inotropic interventions in normal hearts. ${ }^{27-29)}$

Abnormal hearts, though, exhibit different behavior. Hyperthyroidism in the rabbit heart increased the $\mathrm{O}_{2}$ cost of PVA. ${ }^{30)}$ This may be related to the
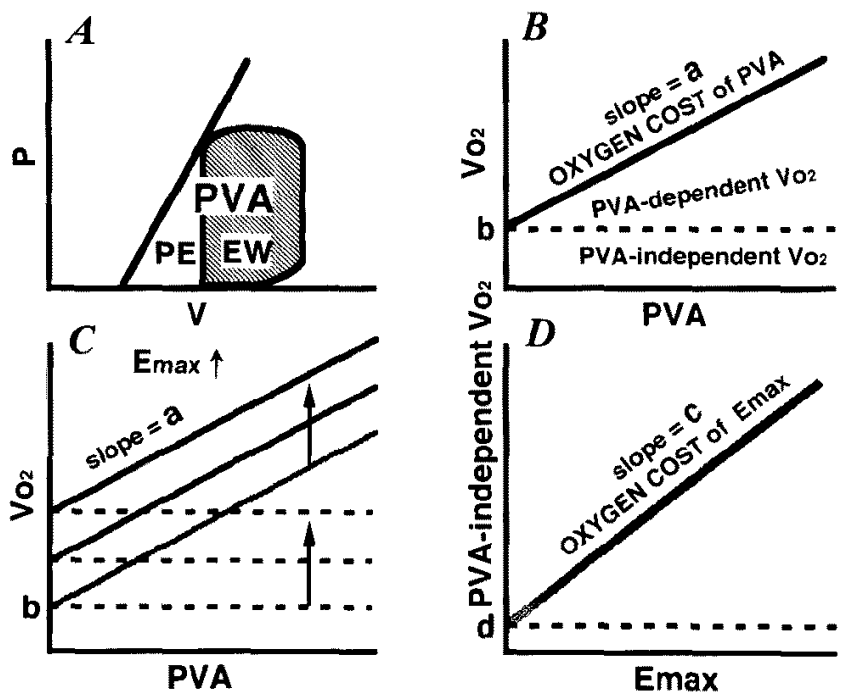

Figure 12. Relations among cardiac oxygen consumption $\left(\mathrm{Vo}_{2}\right)$, PVA and Emax. A: PVA=extemal work (EW) + elastic potential energy (PE). B: Oxygen cost of PVA as the slope a of the $\mathrm{Vo}_{2}-\mathrm{PVA}$ rclation at a given Emax. $\mathrm{C}$ : Vor $-\mathrm{PVA}$ relations at three Emax levels. $\mathrm{VO}_{2}$ intercept $b$ increases with Emax without changes in slope a. D: Oxygen cost of Emax as the slope $\mathrm{c}$ of the PVA-independent $\mathrm{Vo}_{2}$-Emax relation, d=PVA- independent $\mathrm{Vo}_{2}$ at zero Emax. 
massive isomyosin shift from $\beta$ to $\alpha$ type. Such an isomyosin shift was hardly observed in the hypothyroid dog heart, resulting in the absence of an increase in the $\mathrm{O}_{2}$ cost of PVA. ${ }^{31)}$ Acute failing hearts tended to have a slightly decreased $\mathrm{O}_{2}$ cost of PVA, although the underlying mechanism remains to be elucidated. ${ }^{32,33)}$

The $\mathrm{O}_{2}$ cost of Emax increased in stunned myocardium and acidotic myocardium. ${ }^{32,33)}$ This seems to reflect a malfunction of the E-C coupling. We speculated that futile $\mathrm{Ca}^{2+}$ cycling in the sarcoplasmic reticulum and a decreased $\mathrm{Ca}^{2+}$ sensitivity of the contractile machinery underlie the pathophysiology. Thus, we consider that analyses of cardiac energetics by the Emax and PVA concept are helpful to characterize normal and diseased hearts and differentiate their pathophysiology and the underlying mechanisms. ${ }^{27,29)}$

Emax and PVA have been shown to be powerful indices for assessing the mechanoenergetic effects of pharmacologic agents, including the $\mathrm{Ca}^{2+}$ sensitizer EMD $53998,{ }^{34)}$ the plant alkaloid ryanodine ${ }^{35}$ the phosphatase $2,3 \mathbf{B D M}^{36)}$ and a variety of new cardiotonic agents (OPG-8212, UDCG 115 BS, DPI 201-106, milrinone). ${ }^{37,38)}$

\section{Pump function and pressure-volume window}

In addition to its value as an index of contractility, Emax is also a useful measurc for associating ventricular chamber contractile properties with cardiac pump performance. ${ }^{13)}$ Figure 13 (Panel A) shows that stroke volume is defined as the end-diastolic volume minus end-systolic volume. The end-systolic volume

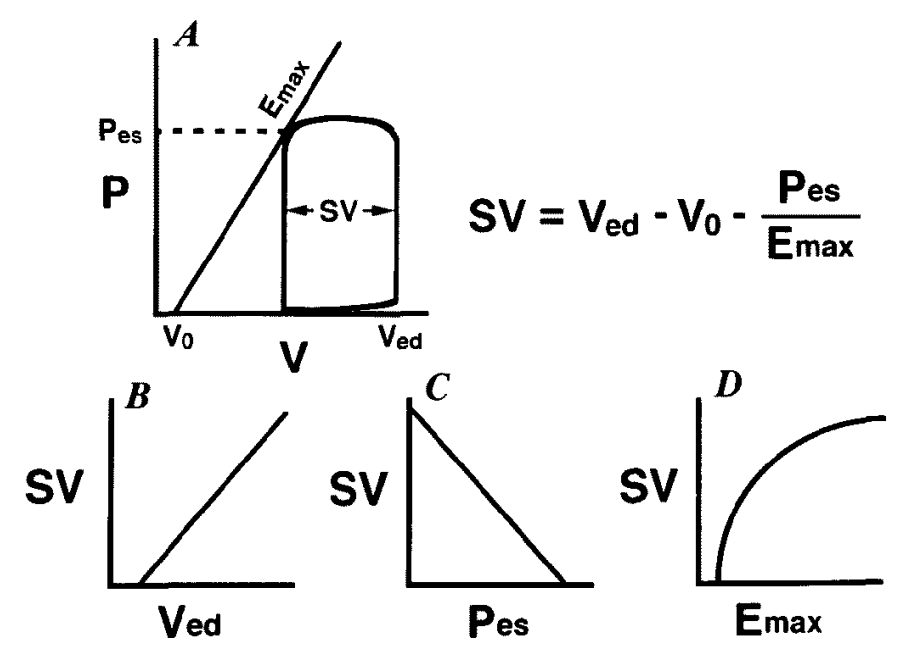

Figure 13. Stroke volume (SV) as a function of end-diastolic volume (Ved), dead volume (Vo), end-systolic pressure (Pes) and contractility (Emax). A: SV, Ved, Vo, Pes and Emax in the P-V diagram. B: SV as an increasing function of Ved. C: SV as a decreasing function of Pes. C: SV as a nonlinearly increasing function of Emax. 
minus Vo is equal to the ratio of end-systolic pressure divided by Emax. These relations are described by the inset equation. This equation, though simple, is meaningful. For example, if preloaded end-diastolic volume alone is changed, stroke volume is an increasing function of end-diastolic volume Ved (Figure 13, Panel B); i.e., it displays the Starling effect. If afterloaded end-systolic pressure alone is changed, stroke volume is a decreasing function of afterload Pes (Figure 13, Panel C). This shows the afterload effect. If Emax alone is changed, stroke volume is a nonlinearly increasing function of Emax (Figure 13, in Panel D). Interestingly, stroke volume is sensitive to changes in Emax in its low range, namely, failing hearts. Stroke volume is insensitive to Emax changes in its normal range (normal hearts). These theoretical predictions are consistent with experimental results.

\section{Ea/Emax ratio}

A new concept, the effective arterial elastance $(\mathrm{Ea})$, was added to the P-V window in 1983 by Kenji Sunagawa. ${ }^{39}$ It is the slope of the line connecting the end-diastolic volume and the end-systolic P-V point (the left upper corner) of the $\mathrm{P}-\mathrm{V}$ loop as shown in Figure 14. Combining Ea with Emax yields very important information on the mechanical and energetic matching of ventriculo-arterial coupling. ${ }^{40)}$

When Ea is comparable to or slightly smaller than Emax, the ventriculoarterial coupling is matched because external work and its efficiency are maximum or near maximum. The situation is found in normal hearts. However, as Ea

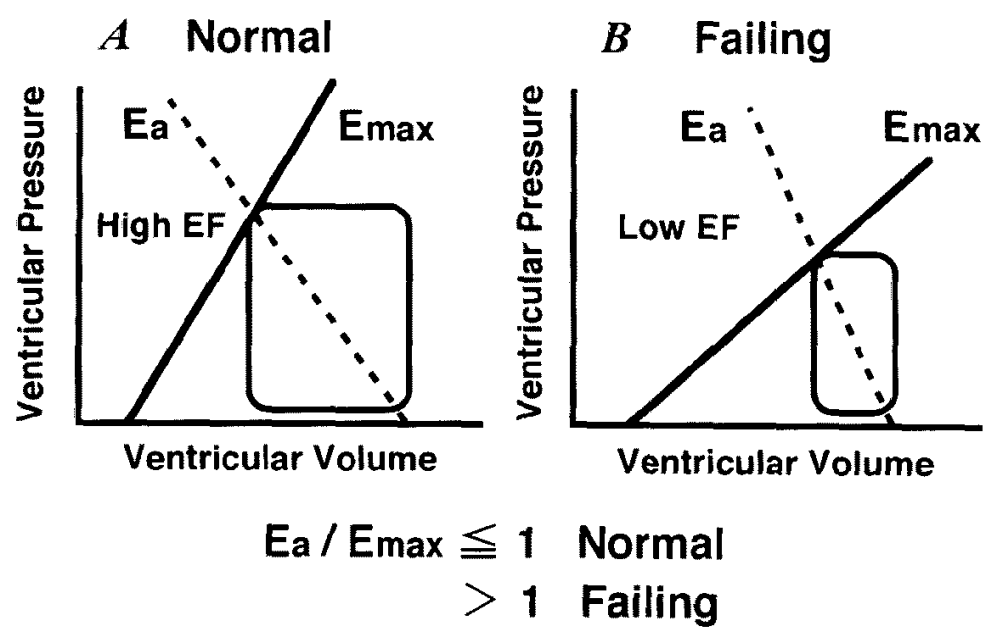

Figure 14. Ventriculo-arterial coupling in terms of Ea/Emax ratio. EF=ejection fraction; $\mathrm{Ea}=$ effective arterial elastance; $E$ max $=$ end-systolic maximum ventricular elastance. A: Fa/Emax ratio is comparable to 1 in normal hearts with a high EF. B; $\mathrm{Ea} / \mathrm{Emax}$ ratio incrcases from 1 as heart failure proceeds with a decreasing $\mathrm{EF}$. 
increases relative to Emax, the degree of matching deteriorates because external work and its efficiency decrease. This situation is found in failing hearts. Therefore, the $\mathrm{P}-\mathrm{V}$ diagram is very helpful for understanding the normality and abnormality of the ventriculo-arterial mechanoenergetic coupling. ${ }^{25,40)}$

The Ea/Emax matching concept has been applied to cardiology. ${ }^{25,41)}$ In a normal heart with an EF above 60\%, Emax was large and comparable to or slightly greater than Ea. In a moderately failing heart with an EF above $40 \%$, Emax decreased and Ea increased with a decreased stroke volume, increasing the $\mathrm{Ea} / \mathrm{Emax}$ ratio. In a severely failing heart with a further decreased EF below $40 \%$, Emax further decreased and the Ea further increased, increasing the Ea/ Emax ratio considerably.

The Ea/Emax ratio is very helpful in the treatment of heart failure because the treatment can be designed to shift the Ea/Emax ratio to approach unity. One method is to decrease Ea by decreasing the heart rate. Another method is to decrease Ea by decreasing the afterload. A third method is to increase Emax. By appropriately combining these three methods, heart failure will be effectively treated so that its ventriculo-arterial coupling is corrected or normalized. Therefore, the $\mathrm{P}-\mathrm{V}$ diagram is very helpful to better understand the ventriculo-arterial coupling and to decide the strategy of cardiological treatment.

\section{Summary and conclusion}

1) Emax can serve as an index of ventricular contractility.

2) Emax is the slope of the ventricular end-systolic P-V relation.

3) Emax represents ventricular end-systolic maximal elastance.

4) Emax characterizes ventricular pump performance.

5) PVA is a measure of the ventricular total mechanical energy generated by contraction.

6) PVA correlates linearly with $\mathrm{Vo}_{2}$. Its slope is the $\mathrm{O}_{2}$ cost of PVA.

7) Changes in Emax shift the $\mathrm{Vo}_{2}$-PVA relation. Its sensitivity is the $\mathrm{O}_{2}$ cost of Emax.

8) $\mathrm{O}_{2}$ costs of PVA and Emax characterize myocardial energetics under various conditions.

9) Emax and PVA, together with Ea (effective arterial elastance), determine cardiac efficiency and load matching.

10) Thus, despite some limitations, the Emax and PVA concepts are useful to characterize abnormalities of diseased hearts.

Therefore, cardiac mechanoenergetic performance of normal and failing hearts has been better understood through the widely opened P-V window. 


\section{ACKNowledgement}

I cordially thank Profs. Masamitsu Oshima (University of Tokyo), Tatsuo Togawa (Tokyo Medical Dental University), Kiichi Sagawa (Johns Hopkins University) and Ishio Ninomiya (National Cardiovascular Center) for their mentorship, and all the coworkers, particularly Dr. Yoichi Goto (National Cardiovascular Center), for their energetic collaboration and constructive discussion.

\section{ReFERENCES}

1. Frank O: Die Grundform des Arteriellen Pulses. Ztschr f Biol 37: 483, 1899

2. Starling EH: Linacre Lecture on the Law of the Heart. Longmans, Green and Co, London, 1918

3. Suga H: Cardiac mechanics and energetics-from Emax to PVA. Front Med Biol Eng 2: 3, 1990

4. Sagawa K, Lie RK, Schaefer J: Translation of "Frank O: Die Grundform des Arteriellen Pulses. Zeitschrift fur Biologie 37: 483-526, 1899" J Mol Cell Cardiol 22: 253, 1990

5. Johnson LR (ed): Essential Medical Physiology. Raven Press, New York, p 183, 1992

6. Patton HD, Fuchs AF, Hille B, Scher AM, Steiner R (eds): Textbook of Physiology, 21st ed, WB Saunders Co, Philadelphia, p 830, 1989

7. Sarnoff SJ, Berglund E: Ventricular function; I. Starling's law of the heart studied by means of simultaneous right and left ventricular function curves in the dog. Circulation 9: 706, 1954

8. Hill AV: The heat of shortening and the dynamic constants of muscle. Proc R Soc London Ser B 126: 136,1938

9. Sonnenblick EH: Force-velocity relations in mammalian heart muscle. Am J Physiol 202: 931,1962

10. Karliner JS, Gault JH, Eckberg D, Mullins CB, Ross J: Mean velocity of fiber shortening; a simplified measure of left ventricular myocardial contractility. Circulation 44: 323, 1971

11. Suga $\mathrm{H}$ : Analysis of left ventricular pumping by its pressure-volume coefficient (in Japanese with English abstract). Jpn J Med Electr Biol Eng 7: 406, 1969

12. Suga $\mathbf{H}$, Sagawa $\mathrm{K}$, Shoukas AA: Load independence of the instantaneous pressure-volume ratio of the canine left ventricle and effects of epinephrine and heart rate on the ratio. Circ Res 32: 314, 1973

13. Sagawa K, Maughan L, Suga H, Sunagawa K: Cardiac Contraction and the Pressure-Volume Relationship. Oxford Univ Press, New York, 1988

14. Suga $\mathbf{H}$ : Total mechanical energy of a ventricular model and cardiac oxygen consumption. Am $J$ Physiol 236: H498, 1979

15. Suga $\mathrm{H}$, Sagawa $\mathrm{K}$ : Instantaneous pressure-volume relationships and their ratio in the excised, supported canine left ventricle. Circ Res 35: 117, 1974

16. Suga $\mathbf{H}$, Kitabatake A, Sagawa K: End-systolic pressure determines stroke volume from fixed enddiastolic volume in isolated canine left ventricle under constant contractile state. Circ Res 44: 238, 1979

17. Suga $H$, Sagawa $K$, Demer L: Determinants of instantaneous pressure in canine left ventricle. Time and volume specification. Circ $\operatorname{Res}$ 46: 256, 1980

18. Suga $\mathbf{H}$, Sagawa $\mathrm{K}$ : End-diastolic and end-systolic ventricular volume clamper for isolated canine heart. Am J Physiol 233: H718, 1977.

19. Sugiura S, Hunter WC, Sagawa K: Long-term versus intrabeat history of ejection as determinants of canine ventricular end-systolic pressure. Circ Res 64: 255, 1989

20. Burkhoff D, Sugiura S, Yue D, Sagawa K: Contractility-dependent curvilinearity of end-systolic pressure-volume relations. Am J Physiol 252: H1218, 1987

21. Suga H: Normalization of end-systolic pressure-volume relation and Emax of different sized hearts. Jpn Circ J 48: 136, 1984

22. Weisfeldt ML, Shoukas AA, Weiss JL, Dashkof N, Conic P, Griffith LSC, Achuff SC, Ducci H, Sagawa $\mathrm{K}$ : Emax as a new contractility index in man (abstract) Circulation 54 (Suppl II): II-31, 1976

23. Grossman W, Braunwald E, Mann T, McLaurin LP, Green LH: Contractile state of the left ventricle in man as evaluated from end-systolic pressure-volume relations. Circulation 56: 845, 1977 
24. Baan J, van der Velde ET, de Bruin HG, Smeenk GJ, Koops J, van Dijk AD, Temmerman D, Senden $\mathrm{J}$, Buis B: Continuous measurement of left ventricular volume in animals and humans by conductance catheter. Circulation 70: 812, 1984

25. Kass DA, Kelly RP: Ventriculo-arterial coupling: concepts, assumptions, and applications. Ann Biomed Eng 20: 41, 1992

26. Suga $H$, Hayashi $T$, Shirahata $M$ : Ventricular systolic pressure-volume area as predictor of cardiac oxygen consumption. Am J Physiol 240: H39, 1981

27. Suga $\mathrm{H}$ : Ventricular energetics. Physiol Rev 70: 247, 1990

28. Suga H, Hisano R, Goto Y, Yamada O, Igarashi Y: Effect of positive inotropic agents on the relation between oxygen consumption and systolic pressure-volume area in canine left ventricle. Circ Res 53: 306,1983

29. Suga $\mathrm{H}$, Goto $\mathrm{Y}$ : Cardiac oxygen costs of contractility (Emax) and mechanical energy (PVA); New key concepts in cardiac energetics, in Recent Progress in Failing Heart Syndrome, ed by Sasayama S, Suga H, Springer-Verlag, Tokyo, p 6l, 1991

30. Goto Y, Slinker BK, LeWinter MM: Decreased contractile efficiency and increased nonmechanical energy cost in hyperthyroid rabbit heart. Circ Res 66: 999, 1990

31. Suga H, Tanaka N, Ohgoshi Y, Saeki, Nakanishi T, Futaki S, Yaku H, Goto Y: Hyperthyroid dog left ventricle has the same oxygen consumption versus pressure-volume area (PVA) relation as euthyroid dog. Heart Vessels 6: 71, 1991

32. Ohgoshi Y, Goto Y, Futaki S, Yaku H, Kawaguchi O, Suga H: Increased oxygen cost of contractility in stunned myocardium of dog. Circ Res 69: 975,1991

33. Hata K, Goto Y, Kawaguchi O, Takasago I, Saeki A, Nishioka I, Suga H: Hypercapnic acidosis increases oxygen cost of contractility in the dog left ventricle. Am J Physiol 1994 (in press)

34. de Tombe PP, Burkhoff D, Hunter. WC: Effects of calcium and EMD-53998 on oxygen consumption in isolated canine hearts. Circulation 86: 1945, 1992

35. Takasago $T$, Goto $Y$, Kawaguchi $O$, Hata K, Saeki A, Nishioka $T$, Suga $H$ : Ryanodine wastes oxygen consumption for $\mathrm{Ca}^{2+}$ handling in the dog heart; A new pathological heart model. J Clin Invest $92: 823$, 1993

36. Yaku H, Slinker BK, Mochizuki T, Lorell BH, LeWinter MM: Use of 2,3-butanedione monoxime to estimate nonmechanical $\mathrm{V}_{2}$ in rabbit hearts. Am J Physiol 265: H834, 1993

37. Futaki S, Goto Y, Ohgoshi Y, Yaku H, Suga H: Similar oxygen cost of myocardial contractility between DPI 201-106 and epinephrine despite different subcellular mechanisms of action in dog hearts. Heart Vessels 7: 8, 1992

38. Hata K, Goto Y, Futaki S, Ohgoshi Y, Yaku H, Kawaguchi O, Takasago T, Saeki A, Taylor TW, Nishioka $T$, Suga H: Mechanoenergetic effects of pimobendan in canine left ventricles; comparison with dobutamine. Circulation 86: 1291, 1992

39. Sunagawa K, Maughan WL, Burkhoff D, Sagawa K: Left ventricular interaction with arterial load studied in isolated canine heart. Am J Physiol 245: H773, 1983

40. Burkhoff D, Sagawa K: Ventricular efficiency predicted by an analytical model. Am J Physiol 250: R1021, 1986

41. Asanoi $H$, Sasayama $S$, Kameyama $T$ : Ventriculoarterial coupling in normal and failing heart in humans. Circ Res 65: 483, 1989 